\title{
CHARACTERIZATION OF TOTALLY UNIMODULAR MATRICES
}

PAUL CAMION

In this paper $A$ will always denote a matrix with entries equal to $1,-1$ or $0 . A$ is totally unimodular if every square submatrix has a determinant equal to $1,-1$ or 0 . A submatrix $A_{J}^{I}$ of $A$ is said to be Eulerian [1] if

$$
\left(\forall_{I} i\right): \sum_{j \in J} A_{j}^{i} \equiv 0 \bmod 2
$$

and

$$
(\forall J j): \sum_{i \in I} A_{j}^{i} \equiv 0 \bmod 2 .
$$

We published in [2] and also in [6] a proof of:

THEOREM 1. $A$ is totally unimodular if and only if every square Eulerian submatrix is singular.

We exposed the proof or the sufficient condition of this theorem as well as the one of Statement 3 at the Seminary on Combinatorial Problems of the Faculté des Sciences de Paris in January 1962. A. Ghouila-Houri gave independently a characterization [3] which could have been deduced however from our results.

$\mathrm{R}$. Gomory gave us recently a new proof of the sufficient condition. His proof, combined with ours, allows us to prove a simpler characterization:

ThEOREM 2. $A$ is totally unimodular if and only if for every (square) Eulerian submatrix $A_{\mathrm{J}}^{I}: \sum_{i \in I ; j \in J} A_{j}^{i} \equiv 0 \bmod 4$.

The following Statements 1 and 2 clearly prove the sufficient condition of Theorem 1.

I. Statement 1 (R. Gomory). If there exists a submatrix $B$ of order $n$ in the matrix $A$ with $|\operatorname{Det}(B)|>2$, there exists a square submatrix $Q$ of $B$ with $|\operatorname{Det}(Q)|=2$.

Proof. Let $D=[B I]$, where $I$ is the unit matrix, and let $\mathfrak{e}$ be the class of all matrices obtained by unimodular row transformations of $D$, with the property: $\left(\forall_{\mathcal{C}} C\right)(\forall j)(\forall i): C_{j}^{i} \in\{1,-1,0\}$ and $C$ contains $n$ different unit column vectors.

Received by the editors June 23, 1964. 
Let $F$ be a matrix in $\mathfrak{e}$ with the greatest number of unit vectors among its first $n$ columns. At least one vector, $F_{k}, k \leqq n$ is not a unit vector. For there cannot be in $\mathfrak{e}$ a matrix of the form $[I G]$, since $|\operatorname{Det}(B)|>2$.

Let us prove that among all possible choices of $n-1$ unit vectors such that $F_{J(r)}=\left[F_{k}, e_{r_{1}}, \cdots, e_{r_{n-1}}\right]$ contains the set $F_{j}, j \in T$ of unit vectors of the first $n$ columns, at least one is such that $F_{J(r)}$ is unimodular.

If this was not true, $F_{J(r)}$ would be singular for each of those choices and to each of those choices would correspond a null coordinate of $F_{k}$.

Let $F_{k}^{i}, i \in S$ be the set of those null coordinates. Thus $F_{T \cup\{k\}}^{S}$ would be a null matrix $(n-|T|) \times(|T|+1)$ and consequently the matrix defined by the first $n$ columns of $F$ would be singular, contrary to the hypothesis that $\operatorname{Det}(B)>2$.

Let $e_{1}$ be the vector which is not in the set $e_{r_{1}}, \cdots, e_{r_{n-1}} ; F_{J(r)}$ (which will be denoted $F_{J}$ for simplicity) being unimodular. $F_{J}^{-1} F$ cannot belong to $\mathcal{C}$, because it has one more unit vector than $F$ in its first $n$ columns. However, $F_{J}^{-1} F$ contains a unit matrix, thus one of the entries of $F_{J}^{-1} F$ is not $1,-1$ or 0 . Let us point out that (after rearrangement of rows and columns),

$$
F_{J}=\left|\begin{array}{cccc}
\epsilon_{1} & 0 & \cdots & 0 \\
\epsilon_{2} & 1 & \cdots & 0 \\
\vdots & \vdots & \cdot & \vdots \\
\epsilon_{n} & 0 & \cdots & . \\
1
\end{array}\right|
$$

where $\epsilon_{1} \in\{1,-1\}$, and

$$
F_{J}^{-1}=\left|\begin{array}{cccc}
\epsilon_{1} & 0 & \cdots & 0 \\
-\epsilon_{2} \epsilon_{1} & 1 & \cdots & 0 \\
\vdots & \vdots & \cdot & \vdots \\
-\epsilon_{n} \epsilon_{1} & 0 & \cdots & .
\end{array}\right| .
$$

$F_{\mathrm{J}}^{-1}$ is the product of elementary matrices, $T_{p}, \cdots, T_{1}$, where $p$ is the number of nonzero $\epsilon_{i}$. Concretely,

$$
T_{1}=\left|\begin{array}{cccc}
\epsilon_{1} & 0 & \cdots & 0 \\
0 & 1 & \cdots & 0 \\
: & \vdots & . & \vdots \\
0 & 0 & \cdots & . \\
0
\end{array}\right|
$$




$$
T_{p}=\left|\begin{array}{rrrr}
1 & 0 & \cdots & 0 \\
0 & 1 & \cdots & 0 \\
\vdots & & & \\
0 & & & \\
-\epsilon_{s} & : & . & . \\
0 & & & \\
\vdots & & & \\
0 & 0 & \cdots & 1
\end{array}\right|
$$

Let $q$ be the smallest integer such that one of the entries of $F^{*}$ $=T_{q} T_{q-1} \cdots T_{1} F$ is not $1,-1$ or 0 .

Clearly, $T_{q}$ is the transformation which adds (subtracts) the first row of $T_{q-1} \cdots T_{1} F$ to (from) the $i$ th row of this matrix. Then, the entries of $F^{*}$ which are not $1,-1$ or 0 are necessarily \pm 2 and they must all be in the $i$ th row. Let $F_{j}^{*_{i}}$ be one of those entries; necessarily, $j \notin J$. Moreover, $T_{q} T_{q-1} \cdots T_{1} F_{k}$ has one more null coordinate than $T_{q-1} \cdots T_{1} F_{k}$ which is precisely $F_{k}^{*_{i}}$. Suppressing $e_{i}$ of $F_{J \cup\{J\}}^{*}$, one finds $F_{J^{\prime}}^{*}$, which has $n-2$ unit column vectors. The submatrix $F_{\{k\} \cup\{\}\}}^{*}(1\} \cup\{i\}$ is necessarily

$$
\left|\begin{array}{rr} 
\pm 1 & \pm 1 \\
0 & \pm 2
\end{array}\right|
$$

thus $\operatorname{Det}\left(F_{J^{\prime}}^{*}\right)= \pm 2$. But $\operatorname{Det}\left(F_{J^{\prime}}^{*}\right)=\operatorname{Det}\left(D_{J^{\prime}}\right)$, since $F_{J^{\prime}}^{*}$ is obtained from $D_{J^{\prime}}$ by a unimodular transformation. As $D=[B I], \operatorname{Det}\left(D_{J^{\prime}}\right)$ is equal to the determinant of one of the square submatrices of $B$.

Statement 2 (R. GOMORY). If for every Eulerian square submatrix $A_{J^{\prime}}^{I^{\prime}}$ of $A, \operatorname{Det}\left(A_{J^{\prime}}^{I^{\prime}}\right)=0$, then, for every square submatrix $A_{J}^{I}$ of $A$, $\operatorname{Det}\left(A_{J}^{I}\right) \equiv 0 \bmod 2$ implies $\operatorname{Det}\left(A_{J}^{I}\right)=0$.

Proof. Let $\mathfrak{C}$ be the class of all square submatrices $A_{J}^{I}$ with $\operatorname{Det}\left(A_{J}^{I}\right)=2 k, k$ nonzero. Let us suppose that $\mathcal{C} \neq \varnothing$. Let $B$ be any matrix of minimum order. There exists a vector $x$ with integral coordinates which are not all zero or even, such that $B x \equiv 0 \bmod 2$, since $\operatorname{Det}(B) \equiv 0 \bmod 2$. Let $B_{K}$ be the set of column vectors of $B$ whose coefficients are the odd coordinates of $x$. These vectors are linearly dependant modulo 2 , thus the determinants of the square submatrices $B_{K}^{I}$ of $B_{K}$ are null modulo 2 .

As they cannot be all zero, $\operatorname{since} \operatorname{Det}(B) \neq 0$, one of them must be even and nonzero.

Since there cannot exist a square matrix $B_{K}^{I}$ in $\mathfrak{C}$ which would be a proper submatrix of $B, K$ is necessarily the set of all column vectors 
of $B$. Thus the row sums of $B$ are even. The same argument applied to $B^{T}$ proves that $B$ is Eulerian, hence singular, which implies that contrary to definition, $\mathfrak{e}$ would contain a singular matrix.

II. We proved in [2] and also in [6] the following statement.

Statement 3. If $A$ is totally unimodular, then to each vector $x$, with $A x \equiv 0 \bmod 2$, corresponds a vector $y$ whose coordinates are $1,-1$ or 0 , with $A y=0$ and $y \equiv x \bmod 2$.

With the help of Statement 3, we shall now prove the necessary condition of Theorem 2. It will be used for proving Statement 4 which with the previous statements will be used to prove the sufficient condition of Theorem 2.

Proof of The NeCESSARy CONDITION OF Theorem 2. For every Eulerian submatrix $A_{J}^{I}$ of the totally unimodular matrix $A$, we have

$$
\left(\forall_{I} i\right): \sum_{j \in J} A_{j}^{i} x_{j} \equiv 0 \bmod 2 ;
$$

where $x_{j}=1$ for each $j$ in $J$. Thus, by Statement 3 , there exists a vector $y$ with coordinates 1 or -1 , for which

$$
\left(\forall_{I} i\right): \sum_{j \in J} A_{j}^{i} y_{j}=0 .
$$

If $y$ has at least one negative coordinate, let $w$ be the vector $y$ where the negative coordinates have been replaced by 1 .

As $A_{J}^{I}$ has an even number of nonzero entries in each column,

$$
\sum_{i \in I_{i} ; \in J} A_{j}^{i} y_{j} \equiv \sum_{i \in I_{;} ; \in J} A_{j}^{i} w_{j_{2}}^{-} \bmod 4
$$

Then, by (2),

$$
\sum_{i \in I ; j \in J} A_{j}^{i} \equiv 0 \bmod 4
$$

Statement 4. Let $A_{J}^{I}$ be a square Eulerian submatrix of a matrix $A$, such that every proper submatrix of $A_{\mathbf{J}}^{I}$ is totally unimodular, then $\sum_{i \in I_{i} ; \in J} A_{j}^{i} \equiv \operatorname{Det}\left(A_{J}^{I}\right) \bmod 4$.

In the case where $A_{J}^{I}$ is singular, the necessary condition of Theorem 2 proves Statement 4 . So, let $A_{J}^{I}$ be nonsingular. As

$$
\sum_{j \in J} A_{J}^{I-\{k\}} x_{j} \equiv 0 \bmod 2,
$$

where $x_{j}=1$, for all $j$ in $J$, there exists a vector $y$ with coordinates 1 or -1 (Statement 3 ), for which 


$$
\sum_{j \in J} A_{J}^{I-\{k\}} y_{j}=0 \text {. }
$$

Let $y^{D}$ be the diagonal matrix where the $j$ th diagonal element is $y_{j}$ and let $\alpha$ be the number: $\sum_{j \in J} A_{j}^{\mathbf{k}} y_{j}$; finally, let $B_{\mathrm{J}}^{I}=A_{\mathrm{J}}^{I} y^{D}$. Then, by (6),

$$
\sum_{i \in I ; j \in J} B_{j}^{i}=\alpha
$$

Since every column of $B_{\mathrm{J}}^{I}$ has an even number of nonzero elements, by the same argument as for the proof of the necessary condition of Theorem 2,

$$
\sum_{i \in I ; j \in J} A_{j}^{i} \equiv \alpha \bmod 4
$$

It suffices now to prove that

$$
\operatorname{Det}\left(A_{J}^{I}\right) \equiv \alpha \bmod 4 .
$$

Let $v$ be the column vector whose coordinates are zero, except the $k$ th which is $\alpha$. Then

$$
y=\left(A_{J}^{I}\right)^{-1} v .
$$

Each element of the $k$ th column of $\left(A_{J}^{I}\right)^{-1}$ must be $1 /|\alpha|$ since for all $j,\left|y_{j}\right|=1$. But each entry of the adjoint of $A_{J}^{I}$ is $1,-1$ or 0 . Thus $\left|\operatorname{Det}\left(A_{J}^{I}\right)\right|=|\alpha|$, which proves (9).

III. Proof of the sufficient condition of Theorem 2. We shall prove that if for every square Eulerian submatrix $A_{\mathrm{J}}^{I}$ of $A$, $\sum_{i \in I, j \in J} A_{J}^{I} \equiv 0 \bmod 4$, then for those matrices $\operatorname{Det}(A)_{J}^{I}=0$. Theorem 1 will end the proof.

Let $\mathfrak{C}$ be the class of Eulerian square submatrices $A_{J}^{I}$ with $\operatorname{Det}\left(A_{J}^{I}\right)$ $\neq 0$. Assume $\mathcal{C}$ is not empty. Let $B$ be any matrix of $\mathfrak{C}$ of minimum order. Then for every square Eulerian proper submatrix $B_{\mathrm{J}}^{I}$ of $B$, $\operatorname{Det}\left(B_{\mathrm{J}}^{I}\right)=0$. By Theorem 1 , this proves that every proper submatrix of $B$ is totally unimodular, and Statement 4 then proves that $\operatorname{Det}(B)$ $\equiv 0 \bmod 4$. On the other hand, applying Statement 1 to $B$, one sees that $|\operatorname{Det} B| \leqq 2$. Then $\operatorname{Det}(B)=0$, and contrary to the hypothesis, $\mathfrak{e}$ would contain a singular matrix.

\section{BIBLIOGRAPHY}

1. C. Berge, Theorie des graphes et ses applications, Dunod, Paris, 1958.

2. P. Camion, Matrices totalement unimodulaires et problèmes combinatoires, Thèse, Université Libre de Bruxelles, Février 1963, Rapport EURATOM EUR 
1632 f. 1964, Presses Académiques Européennes, Bruxelles.

3. A. Ghouila-Houri, Caractérisation des matrices totalement unimodulaires, C. R. Acad. Sci. Paris 254 (1962), 1192-1194.

4. I. Heller, On linear systems with integralvalued solutions, Pacific J. Math. 7 (1957), 1351-1364.

5. W. Tutte, Matroids and graphs, Trans. Amer. Math. Soc. 90 (1959), 527-552.

6. P. Camion, Caractérisation des mutrices unimodulaires, Cahiers Centre Etudes Rech. 5 (1963), no. 4.

EURATOM, ISPRA, ITALY

\section{PRIME RINGS WITH MAXIMAL ANNIHILATOR AND MAXIMAL COMPLEMENT RIGHT IDEALS ${ }^{1}$}

KWANGIL KOH AND A. C. MEWBORN

1. Introduction. Let $R$ be a prime ring with a maximal annihilator right ideal and a maximal complement right ideal. Then there is a division ring $D$ such that either $R$ is isomorphic to a right order in the complete ring of linear transformations of a finite dimensional $D$-space, or for each positive integer $n$ there is a subring $R^{(n)}$ of $R$ which is isomorphic to a right order in the complete ring of linear transformations of an $n$-dimensional $D$-space. This is related to a result of $\mathrm{N}$. Jacobson $[2$, p. 33] and extends a theorem of A. W. Goldie $[1$; Theorem 4.4$]$ that a prime ring with maximum conditions on annihilator right ideals and complement right ideals is a right order in a simple ring with minimum condition on right ideals. $R$ is also isomorphic to a weakly transitive ring of linear transformations of a vector space. This is a generalization of a theorem of $\mathrm{R}$. E. Johnson $[4 ; 3.3]$.

2. We assume throughout that $R$ is a prime ring. The notation $R_{r}^{\Delta}\left(R_{l}^{\Delta}\right)$ is used to denote the right (left) singular ideal of $R$, and $L_{r}^{*}\left(L_{l}^{*}\right)$ is the lattice of closed right (left) ideals of $R$. An $R$-module is uniform if each pair of nonzero submodules has nonzero intersection. A right (left) ideal of $R$ is uniform if it is uniform as right (left) $R$-module. For other definitions and notation see [6].

THEOREM 1. $R$ contains a maximal annihilator right ideal and $a$ maximal complement right ideal if and only if $R_{r}^{\Delta}=(0)$ and $L_{r}^{*}$ is atomic.

Received by the editors July 1, 1964.

1 The authors wish to thank Professor R. E. Johnson for many helpful comments in revising the original manuscript of this paper. 